BULl. AUSTRAL. MATH. SOC.

VOL. 35 (1987) 291-298

\title{
FINITE SOLUBLE GROUPS \\ HAVE LARGE CENTRALISERS
}

\author{
JOHN COSSEY
}

We say that a finite group $G$ has a large centraliser if $G$ contains a non-central element $x$ with $\left|C_{G}(x)\right|>|G|^{\frac{1}{2}}$. We prove that every finite soluble group has a large centraliser, confirming a conjecture of Bertram and Herzog.

Let $C$ denote the class of all finite groups $G$ such that $G$ contains a non-central element $x$ with $\left|C_{G}(x)\right|>|G|^{\frac{3}{2}}$ : we will call a group in $C$ a group with a large centralizer. In a recent paper [2] Bertram and Herzog have presented evidence to support the conjecture that all finite soluble groups are in $C$, extending the results of [1] in which Bertram showed that every finite soluble group $G$ contains an element $x$ with $\left|C_{G}(x)\right|>|G|^{1 / 3}$. The aim of this paper is to show that the conjecture is true. For the remainder of this paper all groups will be finite and soluble.

We will proceed by considering the structure of a minimal soluble group not in $C$; Bertram and Hexzog have obtained a number of restrictions on the structure of groups not in $C$, and in particular have shown that if $G$ is not in $C$, then for some prime $p, G \in D_{p}$, where

Received 22 April, 1986.

Copyright Clearance Centre, Inc. Serial-fee code: 0004-9727/87 $\$ A 2.00+0.00$. 
$D_{p}$ is the class of groups $B$ with the following structure:

$$
F(H)=F\left(H I \times O_{p},(H), O_{p},(H)<Z(H), \text { and } o_{p}(H) \cap Z(H) \neq 1\right. \text {; }
$$

moreover, $O_{p}(H)$ is of class $2, o_{p}(H)^{\prime}<Z(H),\left|O_{p}(H)\right|>|H|^{\frac{2}{2}}$ and $Z(H / F(H))=1$ ([2] Theorem 5 and Corollary 4.1). We note that the same arguments will show that these restrictions on the structure of $o_{p}(G)$ apply to ary noncentral normal $p$-subgroup of $G$. To show that the conjecture is true, it will be enough to prove that if $G \in D_{p}$ then $G \in \mathcal{C}_{O}$, where $\mathcal{C}_{O}$ is the class of groups $H$ which have an element $x$ in $O_{p}(H) \backslash Z(H)$ with $\left|C_{H}(x)\right|>|H|^{\frac{1}{2}}$. We let $G$ be a group of minimal order in $D_{p} \backslash C_{O}$. If the result is true for $G /\left(O_{p},(G) \cap Z(G)\right)$, it is trivial to check that it is true for $G$, and so we must have that $Z(G)$ is a $p$-group and $F(G)=O_{p}(G)$. We set $Z=Z(G)$.

We will need the following facts several times, and will use them without further comment. Let $P, H$ be $p$-subgroups of $G, H \leq O_{p}(G)$, $H$ normalised by $P$ and $h \in H$ such that $P Z / Z \leq C_{G / Z}(h Z)$ : then $\left|P: C_{p}(h)\right| \leq|[H, P]| \leq|Z|$. This is an immediate consequence of the easily verified fact that the map $x+h^{x} h^{-1}(x \in P)$ is a homomorphism from $P$ to $[H, P]$ with kernel $C_{p}(h)$. If $U, V$ are subgroups of $O_{p}(G)$ containing $Z$, then $[U, V]$ (as $\mathbb{Z} G$-module) is an epimorphic image of $(U / Z) \otimes(V / Z)$ (see for example the discussion in Robinson [8] pp. 126-127). If $A$ is an irreducible $G F(p) G$-module of dimension $m>1$, then the multiplicity of the trivial irreducible module in $A \otimes A$ is at most To see this, let $E$ be the algebraic closure of $G F(p)$, and suppose $A^{E}=A_{1} \otimes \ldots \otimes A_{t}$. The $A_{i}$ 's are distinct Galois conjugates (by Theorem 9.21(b) of Isaacs [7]), and so if the multiplicity of the trivial irreducible is to be nonzero, we must have either each $A_{i}$ is self contragredient or the $A_{i}$ 's occur in contragredient pairs. In either case it is clear that the multiplicity is at most 3 . 
We begin by considering the structure of $O_{p}(G)$. Suppose that $o_{p}(G) / Z$ contains two distinct minimal normal subgroups of $G / Z, U / Z$ and $V / Z$. If $|Z|=p^{k},|U / Z|=p^{m},|V / Z| p^{n}$, with $m \leq n$ say, then $|G|^{\frac{1}{2}}<p^{k+m}$. But if $u \in U \backslash Z,\left|C_{U}(u)\right| \geq p^{k+\frac{1}{2} m}$ and $\left|C_{V}(u)\right| \geq p^{k+\frac{3}{2} m}$ (since $[U, V] \neq 1$ only if $U / Z$ and $V / Z$ are contragredient as $G F(p) G$-modules); if follows that $\left.1 C_{U V}(u)\right] \geq p^{k+m}$, a contradiction. Hence $G / Z$ has a unique minimal normal subgroup, $U / Z$ say, with $\mid U / 21=p^{m}$. If $U \neq o_{p}(G)$, let $V / U$ be a minimal normal subgroup of $G / U$ with $V \leq O_{p}(G)$. If $V / Z$ is elementary abelian, we have $[U, V]$ is an epimorphic image of $U / Z \otimes V / Z$ (as $G F(p) G$-module) and so is nontrivial only if $V / U$ is contragredient to $U / Z$ (as $G F(p) G$-modules). It follows that if $u \in U \backslash Z\left|C_{V}(u)\right| \geq p^{k+m}>\left.1 G\right|^{\frac{2}{2}}$, again a contradiction. If $V / Z$ is not elementary abelian, it must be homocyclic with $V / U$ isomorphic to $U / Z$ (as $G F(p) G$-modules). In this case $(U / Z) \otimes(V / Z)$ $\simeq(U / Z) \cdot(V / U)$ and so if $u \in U \backslash Z\left|C_{V}(u)\right| \geq p^{k+2 m-\frac{1}{2} m}>|G|^{\frac{1}{2}}$, a contradiction. Thus we may assume that $o_{p}(G) / Z$ is the unique minimal normal subgroup of $G / Z$.

Let $N=O_{p}(G)^{\prime}$ : then the chief factors of $G$ in $O_{p}(G) / N$ are either central or faithful for $G / O_{p}(G)$ and it follows from the Lemma in Higman [5] that $O_{p}(G) / N=(U / N) \times(2 / N)$ with $U / N$ isomorphic to $O_{p}(G) / Z$ (as $G F(p) G$-module). We set $\quad H=G / O_{p}(G), A=U / N$. We consider the structure of $A$ as a $G F(p) H$-module. Note that $U^{\prime}=N$ and hence $A$ must be self contragredient as $G F(p) H$-module. If $A^{E}=A_{1} \otimes \ldots \otimes A_{t}$, $|A|=p^{m}$, and $|N|=p^{k}$, we have $k \leq t \leq z_{m}$. We can improve this bound slightly. Suppose that $t=b_{2}$; then $A_{1}$ is a two dimensional faithful irreducible $E H$-module, and since $Z(H)=1$ we have by Blichfeldt's Theorem ([3] proposition 11.2) that $A_{1}$ is induced from a one dimensional module $B$ for a subgroup $K$ of index two. We must then have $K$ abelian 
and normal in $H$ : it follows from Gaschutz [4] that $K$ must be cyclic, and from that fact that $Z(H)=1$ that $K$ must be of odd order. If ${ }^{A_{K}}$ is reducible we have $|K|$ divides $p^{3 m}-1$, while if $A_{K}$ is irreducible, we have $E_{G F}(p) K^{(A)}=F$ is a field of order $p^{m}$ normalised by $H$ in $E n d_{G F(p)}(A)$. In this case if $x \in H$ has order two, $x$ acts on $F$ as a Galois automorphism and so in particular the fixed field of $x$ has order $p^{\text {l.m }}$ : it follows that $|K|$ divides $p^{3 m}+1$. Note moreover that the action of $x$ on $A_{1}$ is regular in eithex case and so contains fixed points. It follows that if $\left|O_{p}(G)\right|=p^{m+k+l}$, and $u \in U N$ with $[x, u] \in N,\left|C_{G}(u)\right| \geq 2 p^{m+\ell}$ and so $|G| \geq 4 p^{2 m+2 \ell}$. But $|G|$ $\leq 2 p^{m+k+l}\left(p^{\frac{3}{2} m}+1\right)$ : it is easy to check that the resulting inequality never holds, and hence we must have $t \leq m / 3$.

Note that we have $p^{2 m} \leq|G|<p^{2 m+2 k}$ : set $|G|=p^{2 m+2 \delta}$.

Suppose that for some normal subgroup $X$ of $H$ we have $A_{X}$ non homogeneous : then for some subgroup $I$ of $H$ there is an irreducible $G F(p) I$-module $B$ such that $A=B^{H}$. Set $|H: I|=j, I=J / O_{p}(G)$ : we have $|J|=|G| / j,|B|=p^{m / j}$. If $B=W / N$, and $w \in W N$, then the number of conjugates of $w$ is less than $p^{k+(m / j)}$ and so

$$
\left|C_{J}(w)\right|>|G| p^{-k-(m / j)-\log _{p^{j}}} \geq 2 m+2 \delta-k-(m / j)-\log _{p^{j}} j
$$

It is easy to check that $(m / j)+\log _{p} j \leq 2 m / 3$ (and hence $k+(m / j$ ) $\left.+\log _{p} j \leq m\right)$ unless $m=4$ and $j=2$. But in this case $k=1$ and so $k+(m / j)+\log _{p} j \leq 4$. Thus $\left|c_{G}(w)\right| \geq\left|C_{J}(w)\right|>p^{m+2 \delta} \geq|G|^{\frac{1}{2}}$, a contradiction. We must therefore have $A_{X}$ homogeneous for every normal subgroup $X$ of $H$.

An immediate consequence of this is that every abelian normal subgroup of $H$ must be cyclic, and hence in particular $O_{2}(H)$ 
must be trivial. Since $H$ must then have a nontrivial abelian nomal subgroups of odd order, $A_{1}$ must have even dimension to be self contragredient : it follows that $k \leq m / 4$, and $A$ has even dimensions.

Suppose that $F(H)$ is abelian and hence cyclic. We then have that $H / F(H)$ is cyclic; since $F(H)$ contains no central chief factors it is complemented in $H$, by $L$ say. Since $A_{i F(H)}$ is a direct sum of nonisomorphic irreducibles, it follows that $A_{F(H)}^{E}$ is a direct sum of nonisomorphic irreducibles and thus that $A_{F(H)}$ is irreducible. As above we argue that the action of $L$ on $A$ has fixed points, and $L$ acts as a group of Galois automorphisms on $F(H)$. Thus if $|H / F(H)|=a \leq m / t$ we get $\left.|F(H)| \leq\left(p^{m}-1\right) / p^{m / a}-1\right)$ and then $\left.|G| \leq a p^{m+k+\ell}\left(p^{m}-1\right) / p^{m / a}-1\right)$. But if $x N$ is centralised by $L$, we get $\left|C_{G}(x)\right| \leq p^{m+l} a$, and so we requixe $p^{2 m+2 l} a^{2}<a p^{m+k+l}\left(p^{m}-1\right) /\left(p^{m / a}-1\right):$ using the fact that $a>1$ and $t \leq m / a$, we see this inequality never holds, and so $F(H)$ cannot be abelian.

It then follows from the fact that every characteristic abelian subgroup of $F(H)$ is cyclic that $F(H)$ is the central product of $Z(F(H))$ with a direct product of extraspecial groups of distinct prime power orders (Huppert [6] Satz 3.13.10). Put $Y=Z(F(H)$ ) and let $C=C_{H}(Y)$ : then $C$ is the inextia subgroup of any irreducible constituent of $A_{1 Y}$ and $C \neq H$ : setting $a=|H / C|$, we get $1<a \leq m / 3 t$. If $B$ is an irreducible constituent of $A_{1 C}$ then $B$ is faithful for $F(H)$. If $b=\operatorname{dim} B$ (so that $m=a b t$ ) and $|F(H) / Y|=c^{2}=q_{1}^{2 \alpha_{1}} \ldots q_{r}{ }^{2 \alpha_{r}}$, with $q_{1}, \ldots, q_{r}$ distinct odd primes, then $c \mid b$ and $C / F(H)$ is isomorphic to a subgroup of $\operatorname{sp}\left(2 \alpha_{1}, q_{1}\right) \times \ldots$ $\times \operatorname{sp}\left(2 \alpha_{p}, q_{r}\right) \quad$ (where $\operatorname{sp}\left(2 \alpha_{i}, q_{i}\right)$ is the symplectic group of degree $2 \alpha_{i}$ over $\left.G F\left(q_{i}\right)\right)$. Since $B_{Y}$ and $A_{Y}$ axe homogeneous, the number of distinct Galois conjugates of an irreducible $E Y$-module is at most 
$m / b=a t$, and so $|Y| \leq p^{a t}-1$.

We now obtain a final contradiction by bounding the order of $G$ from above and below and showing that these bounds are incompatible : to do this we need a bound on the order of soluble subgroups of the symplectic groups. Unfortunately the easily accessible bounds seem rather crude, while other bounds (such as those which may be obtained from the classification of maximal subgroups of the symplectic groups) do not seem to improve the bounds significantly in small dimensions. The bound we use will leave us a small number of special cases to consider.

It follows from 2.9.24, 2.8.28, and 2.6.14 of Huppert [6] that a soluble subgroup of $\operatorname{sp}\left(2 \alpha_{i}, q_{i}\right)$ has index greater than $q_{i}{ }^{\alpha_{i}}$ unless $q_{i}^{\alpha_{i}}=3$ and then from 2.9 .13 of Huppert [6] that a soluble subgroup of $\operatorname{Sp}\left(2 \alpha_{i}, q_{i}\right)$ has order less than $q_{i}^{2 \alpha^{2}}$ unless $q_{i}^{\alpha_{i}}=3$. Thus

$$
\begin{aligned}
|C / F(H)| & <3\left(q_{1}^{\alpha_{1}}\right)^{2 \alpha_{1}} \ldots\left(q_{r}^{\alpha_{r}}\right)^{2 \alpha_{r}} \\
& \leq 3\left(q_{1}^{\alpha_{1}} \ldots q_{r}^{\alpha_{r}}\right)^{2 \beta} \\
& =3 c^{2 \beta},
\end{aligned}
$$

where $\quad \beta=\log _{q}(c), q=\min \left\{q_{1}, \ldots, q_{p}\right\}$

We then have that

$$
|G|=p^{m+k+\ell}|Y||F(H) / Y||C / F(H)||H / C| \text {. }
$$

Using the estimates above, we get

$$
|G|<p^{m+k+\ell}\left(p^{a t}-1\right) c^{2} \cdot 3 c^{2 B} \cdot a \text {. }
$$

Note that if $W$ is an irreducible constituent of $B_{F(H)}$ then $W$ is induced from a one dimensional irreducible for an abelian normal subgroup of index $c$, and so there is an element of $W$ (and hence an $x \in U \backslash N$ ) centralised by a subgroup of order $c$. It follows that $\left|C_{G}(x)\right| \geq p^{m+l_{c}}$, 
and so we must have $|G| \geq p^{2 m+2 l} c^{2} ;$ it is clear that for $m$ large enough these estimates will give a contradiction. We show that $p^{m-k-a t+l}-3 c^{2 \beta} \cdot a \geq 0$, a contradiction, except for a small number of cases which we deal with individually. Note that $m \geq 6$ and $m$ is divisible by an odd prime.

Suppose that $p>2$. We have, since $p \geq 3$ and $q \geq 3$, that $p^{m-k-a t+l}-3 a c^{2 \beta} \geq 3^{m-k-\alpha t}-3 a c^{2 \log _{3} c}$. If $a t>2$, we have $a t \leq m / 3$, $a \leq m / 3, k \leq m / 6$, and since $c$ is odd, $m / c$ is even, giving $c \leq m / 4$. We then get $3^{m-k-a t}-3 a e^{2 \log _{3} c} \geq 3^{m / 2}-3(m / 3)(m / 4)^{\log _{3}(m / 4)}$, and it is not difficult to show that $3^{m / 2}-3(m / 4)^{2 \log _{3}(m / 4)}>0$ for $m \geq 6$. If $a t=2$, we have $a=2, t=1$ (and so $k=1$ ), and then $3^{m-k-a t}-3 a c^{2 \log _{3} c} \geq 3^{m-3}-3(m / 2)^{1+2 \log _{3}(m / 2)}$; this also is positive for $m \geq 10$, If $m=6$, then the result follows from the facts that $p \geq 5$, $\ell=0$, and $C / F(H)$ is a subgroup of $S L(2,3)$.

Suppose then that $p=2$. If $c=3$, then $b=6$, being the dimension of a faithful irreducible module for a non abelian group of order 27 over $G F(2)$. Thus $m \geq 12, a \leq a t \leq m / 6$, and the result follows from the fact that $2^{(2 m / 3)-1}-9 m$ is positive for $m \geq 12$. Thus suppose that $c \geq 5$. If $a t>2$, we have $a \leq m / 5$, at $\leq m / 5$, $k \leq m / 10$, and $c \leq m / 4$ : it then follows that $2^{m-k-a t+\ell}-3 a c^{2 \beta}$ $\geq 2^{7 m / 10}-3(m / 5)(m / 4)^{2 \log _{3}(m / 4)}$. It is then easy to check that this is positive for $m \geq 10$, as required. If at $=2$, then $t=k=1$, $\ell=0, a=2$, and $c \leq m / 2$ : we then get $2^{m-1}-18(m / 2)^{2 \log _{3}(m / 2)} \geq 0$ for $m \geq 18$. This leaves us $m=10$ and 14 to consider. Using the formula (i) for $|G|$ and the fact that $C / F(H)$ is a subgroup of $S p(2,5)$ for $m=10$ and of $s p(2,7)$ for $m=14$ we see that neither of these cases can arise.

This establishes the truth of the conjecture. 
The final part of this proof is rather messy : the bounds used are vexy sensitive to small changes in the estimates for $t, k, a, c$. This version involves the smallest number of special cases of those I tried. The testing of the corresponding bounds was greatly facilitated by the use of ANU Graph, a graph drawing package developed by Neville Smythe and Martin Ward.

\section{References}

[1] E.A. Bertram, "Large centralizers in finite soluble groups" Israel J. Math. 47 (1984), 335-344.

[2] Edward A. Bertram and Marcel Herzog, "Finite groups with large centralizers", Bull. Austral. Math. Soc. 32 (1985), 399-414.

[3] C.W. Curtis and I. Reiner, Methods of Representation Theory, Vol. 1. (Wiley-Interscience, New York, 1981.

[4] Wolfgang Gaschütz, "Endliche Gruppen mit treuen absolutirreduziblen Darstellungen", Math. Nachr. 12 (1954), 253-255.

[5] Graham Higman, "Complementation of abelian normal subgroups", Publ. Math. Debrecen. 4 (1956), 455-458.

[6] B. Huppert, Endliche Gruppen I, Grundlehren Math. Wiss. 137 (Springer-Verlag, Berlin, Heidelberg, New York, 1967).

[7] I.M. Isaacs, Character Theory of Finite Groups, (Academic Press, New York, San Francisco, London, 1976).

[8] Derek J.S. Robinson, A course in the theory of groups, Graduate Texts in Math. 80 (Springer-Verlag, Berlin, Heidelberg, New York 1982).

\footnotetext{
Department of Mathematics, Faculty of science, The Australian National University, G.P.O. Box 4, Canberra, 2601 Australian Capital Territory, Australia.
} 